\title{
Estrutura e composição de um trecho de Mata Atlântica no Parque Estadual das Fontes do Ipiranga, São Paulo, SP, Brasil ${ }^{1}$
}

\author{
Murilo Rondoni Tanus ${ }^{2,3}$, Mayara Pastore ${ }^{2}$, Rosangela Simão Bianchini² e Eduardo Pereira Cabral Gomes ${ }^{2}$
}

Recebido: 7.12.2011; aceito: 16.03 .2012

\begin{abstract}
Structure and composition of an Atlantic Forest path in the "Parque Estadual das Fontes do Ipiranga", São Paulo, São Paulo State, Brazil). Structure and composition of a patch of Atlantic Forest in São Paulo plateau was studied in an area of 5 ha in 30 circular plots of $50 \mathrm{~m}^{2}$ (3.99 m radius) at 5, 15 and $45 \mathrm{~m}$ from the edge, distributed in 10 plots by distance. Woody plants over $2.5 \mathrm{~m}$ in height were sampled. Myrtaceae and Lauraceae with 12 species and Rubiaceae with 11, were the richest families in species number. The floristic diversity $\left(\mathrm{H}^{\prime}\right)$ of the sampled area was 4.06. The main botanical families of the São Paulo plateau are represented in the sampled area and this site has an high diversity and species richness compared to other forest parts of the Great São Paulo City, although the high density of some pioneer species such as Alchornea sidifolia Müll. Arg. can show forest tracts disturbed by fragmentation. Management actions for conservation are suggested.
\end{abstract} Key words: diversity, forest fragments, urban forest

RESUMO - (Estrutura e composição de um trecho de Mata Atlântica no Parque Estadual das Fontes do Ipiranga, São Paulo, SP, Brasil). A estrutura e composição de um trecho de Mata Atlântica no planalto paulista foi estudada em uma área de 5 ha em 30 parcelas circulares de $50 \mathrm{~m}^{2}$ (3,99 m de raio) a 5, 15 e $45 \mathrm{~m}$ da borda, distribuídas em 10 parcelas por distância. Amostraram-se os indivíduos lenhosos com mais de 2,5 m de altura. Myrtaceae e Lauraceae, com 12 espécies e Rubiaceae com 11 foram as famílias mais ricas em espécies. A diversidade florística (H’) da área amostrada foi 4,06. As principais famílias botânicas do planalto paulista estão bem representadas na amostra e a área apresenta alta diversidade e riqueza de espécies, quando comparadas a outros trechos florestais da Grande São Paulo, embora a alta densidade de algumas espécies pioneiras, como Alchornea sidifolia Müll. Arg., possa evidenciar trechos perturbados pela fragmentação. Ações de manejo para conservação são sugeridas.

Palavras-chave: diversidade, floresta urbana, fragmentos florestais

\section{Introdução}

A Floresta Atlântica representa uma região heterogênea, composta por formações distintas com elevada riqueza de espécies e diversidade florística, superando em alguns trechos, às observadas em florestas equatoriais amazônicas (Joly et al. 1991, Thomas et al. 1998, Tabarelli \& Mantovani 1999). No Estado de São Paulo há uma grande tradição de estudos descritivos fitossociológicos e levantamentos florísticos (Barros et al. 2002) em áreas de remanescentes florestais, possibilitando a identificação de padrões estruturais e de distribuição da diversidade, nesses trechos e suas relações florísticas com outros fragmentos (Dislich et al. 2001,
Ogata \& Gomes 2006). Esses trabalhos permitem analisar o grau de similaridade florística entre florestas de distintas regiões do Estado, como entre áreas de floresta do planalto paulistano (Rossi 1994, Groppo Jr. 1999), áreas de Mata Atlântica do litoral (Barros et al. 1991, Mamede et al. 2001) e florestas do interior do Estado (Leitão Filho 1992).

O Parque Estadual das Fontes do Ipiranga (PEFI), local do presente estudo, juntamente com as florestas localizadas na Grande São Paulo, mostra maior similaridade, em sua composição, com as florestas Atlânticas mais úmidas do que com as florestas do interior (estacionais) do Estado (Gomes 1992, 1998, Aragaki 1997) e pertence às florestas nativas que recobrem o Planalto Atlântico do Estado de São Paulo.

1. Parte da Dissertação de Mestrado do primeiro Autor

2. Instituto de Botânica, Av. Miguel Stéfano 3687, Caixa Postal 68041, 04045-972 São Paulo, SP, Brasil

3. Autor para correspondência: murilort@gmail.com 
Dentre essas formações de florestas atlânticas, muitas são fragmentos florestais urbanos, como a Mata da Cidade Universitária (CUASO), o Parque Santo Dias, a Mata do Parque Alfredo Volpi, o PEFI, entre outras. Essas são, a priori, áreas diminutas com acentuado isolamento em decorrência da ocupação antrópica (Santin 1999). A redução da área e a mudança na forma de trechos florestais, causados pela fragmentação florestal, proporcionam mudanças microclimáticas que favorecem o estabelecimento de espécies pioneiras oportunistas, que levam a um declínio na riqueza e diversidade de fragmentos florestais (Turner \& Corlett 1996).

Nesse contexto, a urbanização intensifica essas modificações e torna suas implicações mais graves, podendo levar, inclusive, a processos de extinções biológicas (Peccinini \& Pivello 2002). Todavia a utilização do PEFI como área de desenvolvimento de atividades ambientais, pesquisa e preservação permanente dos ecossistemas naturais presentes, como unidade de conservação, pode minimizar a ação de alguns fatores perturbadores, como incêndios, invasões e extrações seletivas (Santin 1999, Barbosa et al. 2002).

As florestas pertencentes ao domínio da Mata Atlântica são uma das mais ameaçadas pela fragmentação, dentre todas as florestas brasileiras, especialmente suas matas de planalto, como as encontradas no PEFI (Viana et al. 1997), um dos poucos remanescentes urbanos deste tipo florestal.

Assim, os estudos de fitossociologia tornam-se primordiais à caracterização das comunidades vegetais e à melhor interpretação da distribuição das espécies em determinada área florestal (Felfili \& Rezende 2003), possibilitando sua utilização em programas públicos de recuperação de áreas degradas e de compensação ambiental.

No presente trabalho nosso objetivo foi descrever a estrutura do componente arbóreo-arbustivo de um trecho de Floresta Atlântica do PEFI ao longo do gradiente borda-interior.

\section{Material e métodos}

Área de estudo - A área onde foram realizadas as coletas dos dados localiza-se no PEFI, município de São Paulo, Estado de São Paulo. Situado no planalto paulista e inserido no domínio da Mata Atlântica, possui espécies típicas da Floresta Ombrófila Densa e representantes da Floresta Estacional Semidecidual (Nastri et al. 1992, Barros et al. 2002). O PEFI situa-se em ambiente intensamente urbanizado, formando uma "ilha florestal" inserida na metrópole paulistana
(Fernandes et al. 2002). O Parque localiza-se entre os paralelos $23^{\circ} 38^{\prime} 08^{\prime}$ 'S e $23^{\circ} 40^{\prime} 18^{\prime \prime}$ S e meridianos $46^{\circ} 36^{\prime} 48^{\prime \prime} \mathrm{W}$ e $46^{\circ} 38^{\prime} 00^{\prime \prime} \mathrm{W}$, em área total próxima de 527 ha, com altitudes que variam entre 770 e $825 \mathrm{~m}$ (Barbosa et al. 2002). Possui clima temperado Cwb, segundo a classificação de Köppen, com temperatura média anual de $19,1{ }^{\circ} \mathrm{C}$ e precipitação média de $1.540 \mathrm{~mm}$ por ano (Santos \& Funari 2002). O solo de maior ocorrência na área é o Latossolo Vermelho-Amarelo.

O PEFI tem fundação oficial datada de 1969, embora seus limites tenham sido demarcados desde o final do século XIX. A área era de propriedade de fazendeiros e agricultores e apenas após a desapropriação total do local, em 1917, teve início a recuperação da vegetação. Trata-se, portanto, de uma vegetação secundária, mas que está há quase 100 anos protegida e em processo de recuperação (Barbosa et al. 2002, Barros et al. 2002).

A área do estudo (figura 1), possui 5 ha e apresenta, a grosso modo, forma triangular com o maior lado voltado para o norte e nenhum ponto no fragmento encontra-se a mais de $100 \mathrm{~m}$ de uma das bordas. Foi escolhida uma face intermediária, quanto à condição de luz, a sudoeste para a implantação das parcelas e coleta dos dados. Esta face apresenta na região oposta ao trecho de mata, uma alameda asfaltada e vegetação herbácea, onde localizam-se três lagos artificiais. $\mathrm{O}$ maior lado possui, em parte de seu entorno, um arboreto e a face leste do trecho é limitada por outra alameda asfaltada, que separa, brevemente, a área de estudo da continuidade da vegetação nativa do PEFI.

Procedimento de campo - As coletas foram realizadas no segundo semestre de 2010 e terminaram no início de 2011. Todos os indivíduos lenhosos com altura $\geq 2,5 \mathrm{~m}$ foram amostrados em parcelas circulares de $50 \mathrm{~m}^{2}(3,99 \mathrm{~m}$ de raio) a cada uma das seguintes distâncias em relação à borda: $5 \mathrm{~m}$ (partindo do centro da circunferência), $15 \mathrm{~m}$ e $45 \mathrm{~m}$ (10 parcelas por distância), totalizando uma área de amostragem de $1500 \mathrm{~m}^{2}$. Como acima exposto, nenhum ponto do fragmento encontra-se a mais de $100 \mathrm{~m}$ de uma das bordas, podendo ser considerado, neste caso, que as parcelas a $45 \mathrm{~m}$ dos limites do trecho são fisionomicamente representativas do interior do fragmento e àquelas a $15 \mathrm{~m}$ caracterizam uma situação intermediária, entre estas e as de borda (Tanus 2011). No presente trabalho nos concentramos, especificamente, na caracterização florística e estrutural do trecho estudado, reservando para um próximo artigo a análise do efeito de borda. 


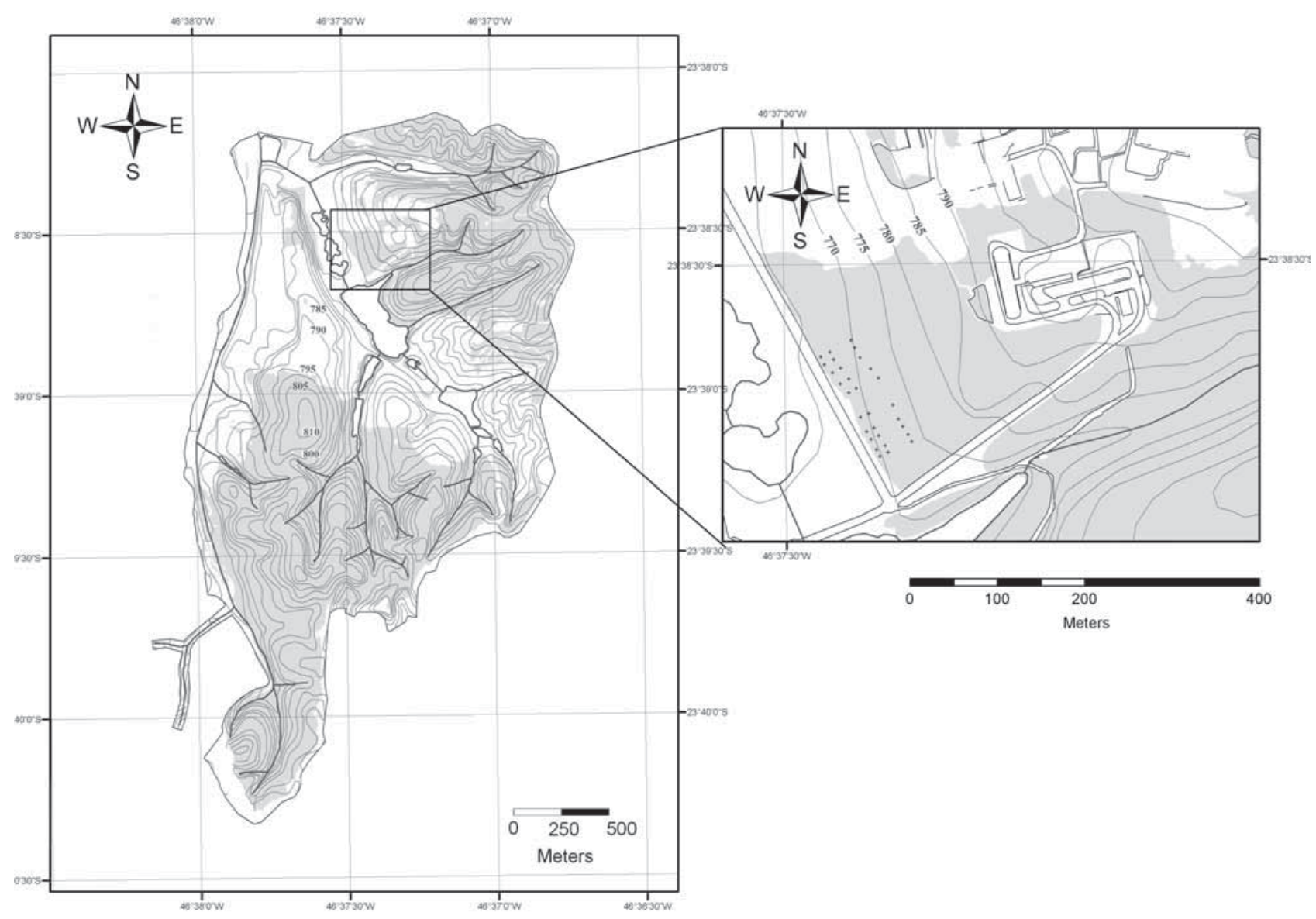

Figura 1. Mapa do Parque Estadual das Fontes do Ipiranga, São Paulo, SP, Brasil. Em destaque, à direita, localização de parte da área escolhida para a coleta de dados, com as 30 parcelas $(+)$ utilizadas no trecho de floresta estudado (C). Regiões em cinza ( $\square$ ) indicam cobertura por mata nativa.

Figure 1. Map of Fontes do Ipiranga State Park, São Paulo, São Paulo State, Brazil. Featured in figure, location of the area chosen for data collection with the 30 sample plots $(+)$ used in the patch of forest studied. Regions in Gray( indicate coverage by native forest.

Os espécimes amostrados foram mensurados quanto à altura e PAP (perímetro a 1,30 $\mathrm{m}$ do solo), os indivíduos que apresentaram mais de um caule a essa altura tiveram os perímetros de cada caule registrados separadamente. A presença de indivíduos mortos e de lianas foi registrada. Lianas não foram coletadas e ramos com estruturas vegetativas e/ou sexuais foram coletados e herborizados para identificação. As identificações foram confirmadas por meio de comparações com no herbário do Instituto de Botânica (SP). Nomes e números de coletas das exsicatas consultadas são apresentados na tabela 1 . O material coletado não foi depositado no herbário SP, devido a falta de espaço e, principalmente, por não se tratar de nenhuma espécie nova para a flora do PEFI, já publicada. O material coletado encontra-se alocado no Núcleo de Pesquisas em Ecologia do Instituto de Botânica.

Análise dos dados - Todos os indivíduos foram identificados com o auxílio de literatura especializada, com destaque para os volumes da revista Hoehnea, que contêm os artigos com as chaves dicotômicas das espécies encontradas em trabalhos realizados no Parque (Melhem et al. 1981). Foram feitas comparações com exsicatas da coleção do herbário e pesquisadores taxonomistas especialistas foram consultados, para maiores esclarecimentos.

A listagem dos nomes das espécies foi construída de acordo com o sistema de classificação APG III (2009) para circunscrição das famílias e, para conferir a validade da nomenclatura das espécies, a Lista de Espécies da Flora do Brasil 2010 (Forzza et al. 2010), disponibilizada no site http://floradobrasil.jbrj. gov.br/2010. Utilizou-se o programa Fitopac versão 2.1.2 (Shepherd 2010) para o cálculo do índice de diversidade de Shannon e descritores quantitativos (Mueller-Dombois \& Ellenberg 1974).

Para realizar a comparação dos dados obtidos no presente trabalho com aqueles de outras regiões 
próximas ao PEFI, procedeu-se à equiparação dos valores de DAP.

\section{Resultados}

Foram amostrados 1.080 indivíduos, com o registro de 99 indivíduos mortos em pé. Os indivíduos distribuíram-se por 118 espécies, 80 gêneros e 35 famílias botânicas; três espécies foram identificadas apenas em nível de gênero e quatro em nível de família, em uma área amostral de 0,15 ha. As formas de vida foram representadas por $23,5 \%$ de lianas, $3,2 \%$ de palmeiras e as demais por indivíduos arbustos ou árvores.

As famílias Myrtaceae e Lauraceae apresentaram o maior número de espécies, com 12 espécies cada uma, seguidas por Rubiaceae, 11 espécies, Melastomataceae, 10 e Fabaceae nove espécies. Os gêneros de maiores riquezas florísticas foram: Miconia (sete); Solanum e Ocotea (cinco); Psychotria (quatro) e Mollinedia e Dalbergia (três). Os gêneros restantes (74) foram representados por duas ou uma espécie.

A área basal do trecho florestal estudado foi de 31,71 $\mathrm{m}^{2} \mathrm{ha}^{-1}$, com Alchornea sidifolia Müll. Arg. $\left(10,5 \mathrm{~m}^{2} \mathrm{ha}^{-1}\right)$, Anadenanthera colubrina (Vell.) Brenan (3,59 $\left.\mathrm{m}^{2} \mathrm{ha}^{-1}\right)$, Lauraceae sp. $1\left(2,1 \mathrm{~m}^{2} \mathrm{ha}^{-1}\right)$, Ocotea puberula (Rich.) Nees $\left(1,61 \mathrm{~m}^{2} \mathrm{ha}^{-1}\right)$ e Ecclinusa ramiflora Mart. $\left(1,51 \mathrm{~m}^{2} \mathrm{ha}^{-1}\right)$, perfazendo $60,9 \%$ do total da área basal da comunidade vegetal. As 10 espécies com maior Valor de Importância (VI) representaram $40 \%$ do VI total, com destaque para Alchornea sidifolia Müll. Arg., Guarea macrophylla Vahl, Syagrus romanzoffiana (Cham.) Glassman, Casearia sylvestris Sw. e Ocotea puberula (Rich.) Nees (tabela 1).

A densidade total na área amostrada foi de 5.506 indivíduos ha ${ }^{-1}$, excluindo-se os indivíduos mortos ou 1.926 indivíduos ha ${ }^{-1}$, quando considerados indivíduos com DAP $\geq 4,7 \mathrm{~cm}$. Alchornea sidifolia Müll. Arg. foi a espécie que mais contribuiu para a densidade absoluta (460 indivíduos ha-1), seguida por Guarea macrophylla Vahl (306,7 indivíduos ha-1), Cupania oblongifolia Mart. (260 indivíduos ha-1), Casearia sylvestris Sw. (240 indivíduos ha-1) e Ocotea puberula (Rich.) Nees (186,7 indivíduos ha-1). Essas cinco espécies somam $26,4 \%$ da densidade relativa. As espécies com maior dominância foram Alchornea sidifolia Müll. Arg. $\left(9,58 \mathrm{~m}^{2} \mathrm{ha}^{-1}\right)$, Syagrus romanzoffiana (Cham.) Glassman $\left(4,02 \mathrm{~m}^{2} \mathrm{ha}^{-1}\right)$, Guarea macrophylla Vahl $\left(2,46 \mathrm{~m}^{2} \mathrm{ha}^{-1}\right)$, Anadenanthera colubrina (Vell.) Brenan $\left(2,43 \mathrm{~m}^{2} \mathrm{ha}^{-1}\right)$ e Lauraceae 1 $\left(2,09 \mathrm{~m}^{2} \mathrm{ha}^{-1}\right)$, sendo responsáveis por $56,8 \%$ da dominância relativa total.

O número de espécies raras (sensu Martins 1991), ou seja, aquelas que foram amostradas por um único indivíduo, foi de 35 ou $29,6 \%$ do total de espécies, correspondendo a $3,2 \%$ do número de indivíduos amostrados. A diversidade florística $\left(\mathrm{H}^{\prime}\right)$ da área amostrada foi 4,06 e a equabilidade de Pielou (J') 0,85.

\section{Discussão}

A densidade de 1.926 indivíduos ha- ${ }^{-1}$ assemelha-se às densidades observadas em outros trechos florestais da Grande São Paulo, que geralmente variam de 1.488 a 2.010 indivíduos ha-1 (Struffaldi-De-Vuono 1985, Nastri et al. 1992, Dislich et al. 2001) em amostragens com até 500 indivíduos com DAP $\geq 4,7 \mathrm{~cm}$.

A alta densidade relativa de lianas pode refletir o histórico de ocupação das áreas do PEFI. Esse Parque abriga instituições de pesquisa, serviços e lazer, com grande circulação humana, tornando as áreas de florestas nativas da região alvos de perturbações diretas ou indiretas, decorrentes de processos de fragmentação e de atividades realizadas em seu entorno e interior (Pivello \& Peccinini 2001). Esta fragmentação provoca mudanças microclimáticas que favorecem o estabelecimento de plantas resistentes, como muitas lianas, em detrimento das espécies de árvores não pioneiras (Nascimento \& Laurance 2006, Michalski et al. 2007).

Quando comparado com levantamentos feitos na Grande São Paulo e proximidades (Ogata \& Gomes 2006) as famílias botânicas com maior número de espécies e que contribuem para a diversidade são praticamente as mesmas Myrtaceae, Lauraceae, Rubiaceae, Melastomataceae e Fabaceae, corroborando os resultados de Gentry (1988) ao observar a previsibilidade, em escalas geográficas amplas, do táxon família, o que não ocorre no nível de espécie.

Não há uma clara relação entre riqueza florística e densidade de indivíduos, quando comparadas áreas florestais da Grande São Paulo. No entanto, de um modo geral, os trechos com as maiores densidades são os que possuem o maior número de espécies, como registrado no presente estudo, para indivíduos com DAP $\geq 4,7 \mathrm{~cm}$, densidade de1.926 indivíduos ha-1 que somaram 81 espécies, ou em um trecho de Mata da Serra da Cantareira (Tabarelli \& Mantovani 1997) para indivíduos com DAP $\geq 3 \mathrm{~cm}$, densidade de 2.350 indivíduos ha ${ }^{-1}$, com 84 espécies. Já em trecho da Mata da Reserva da Cidade Universitária Armando 


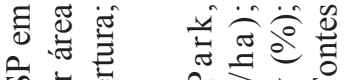

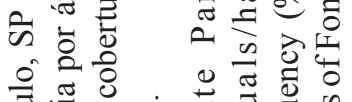

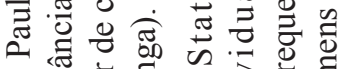

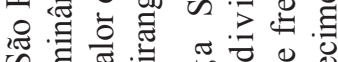

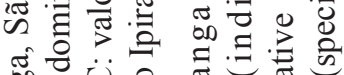

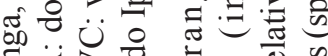

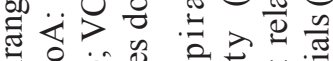
: ○

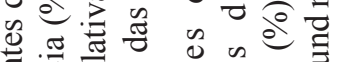
司击

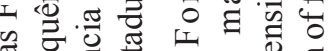

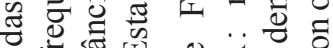

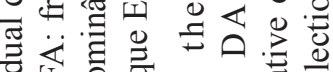
可造总

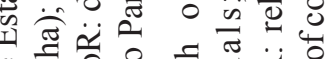

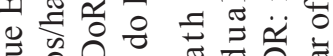
을유

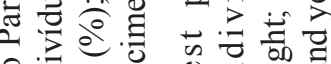
을 ฮี

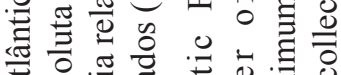

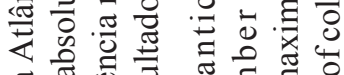

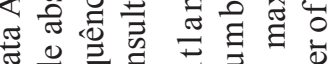

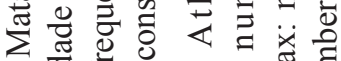
ष्ठ

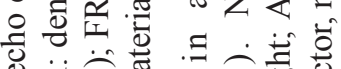

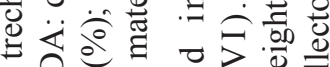

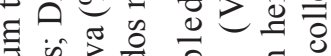

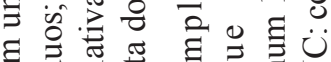

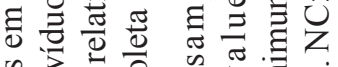

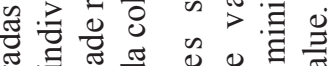

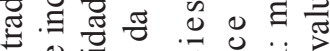

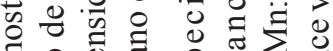

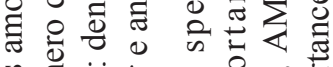

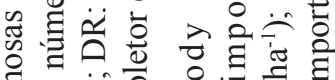

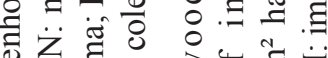

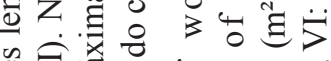
氖氞

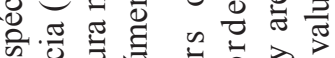

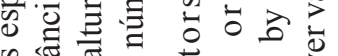

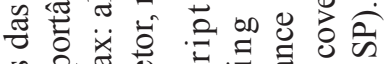

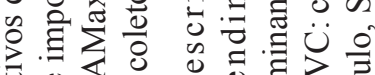

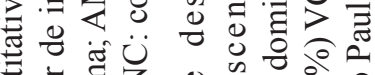

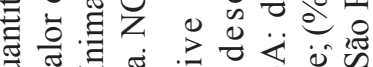

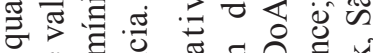

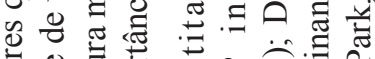

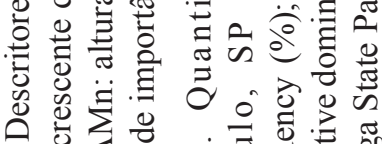

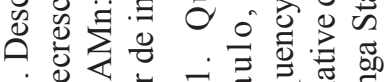

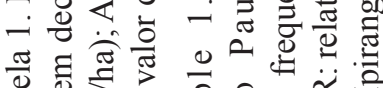

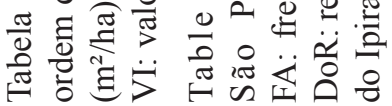

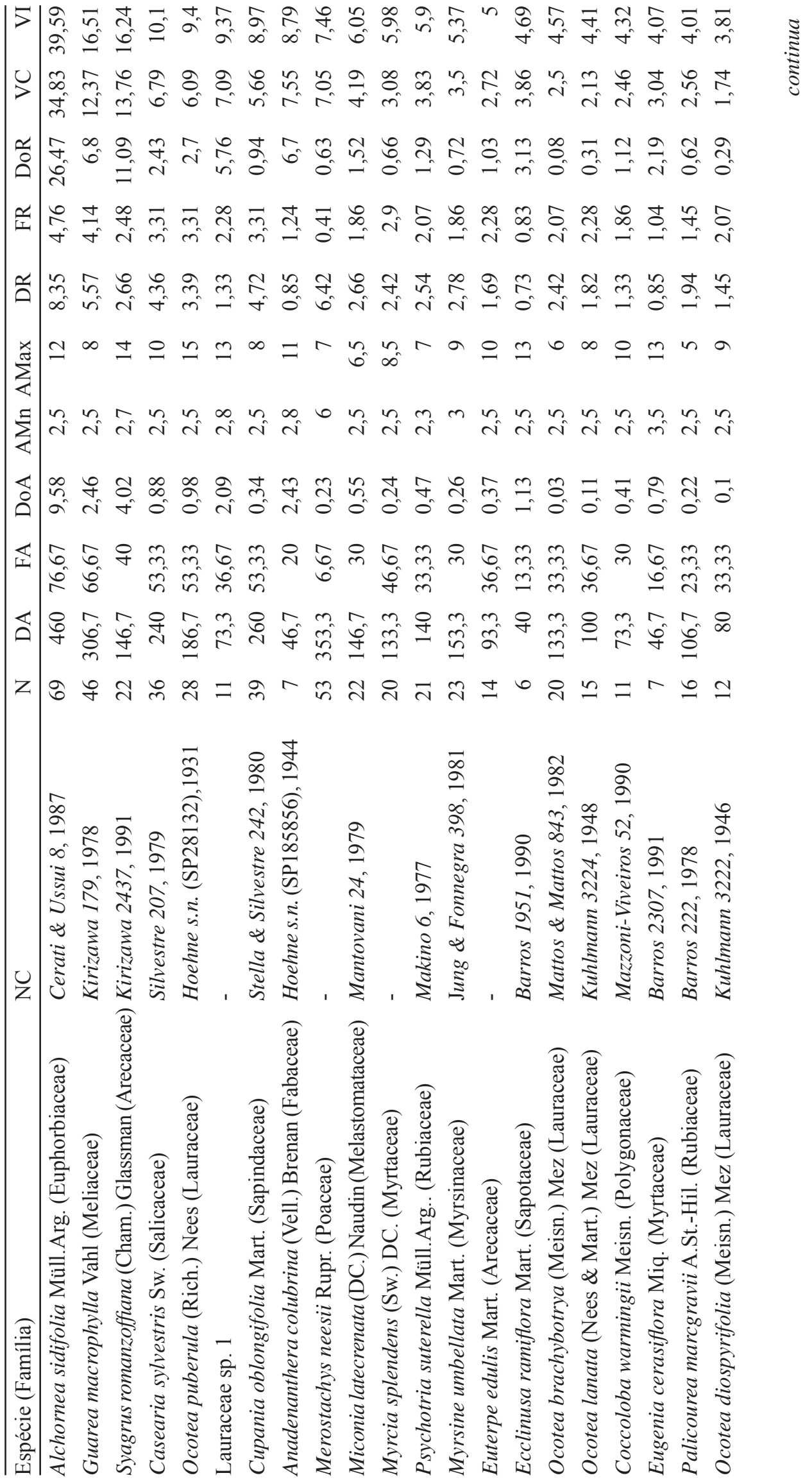




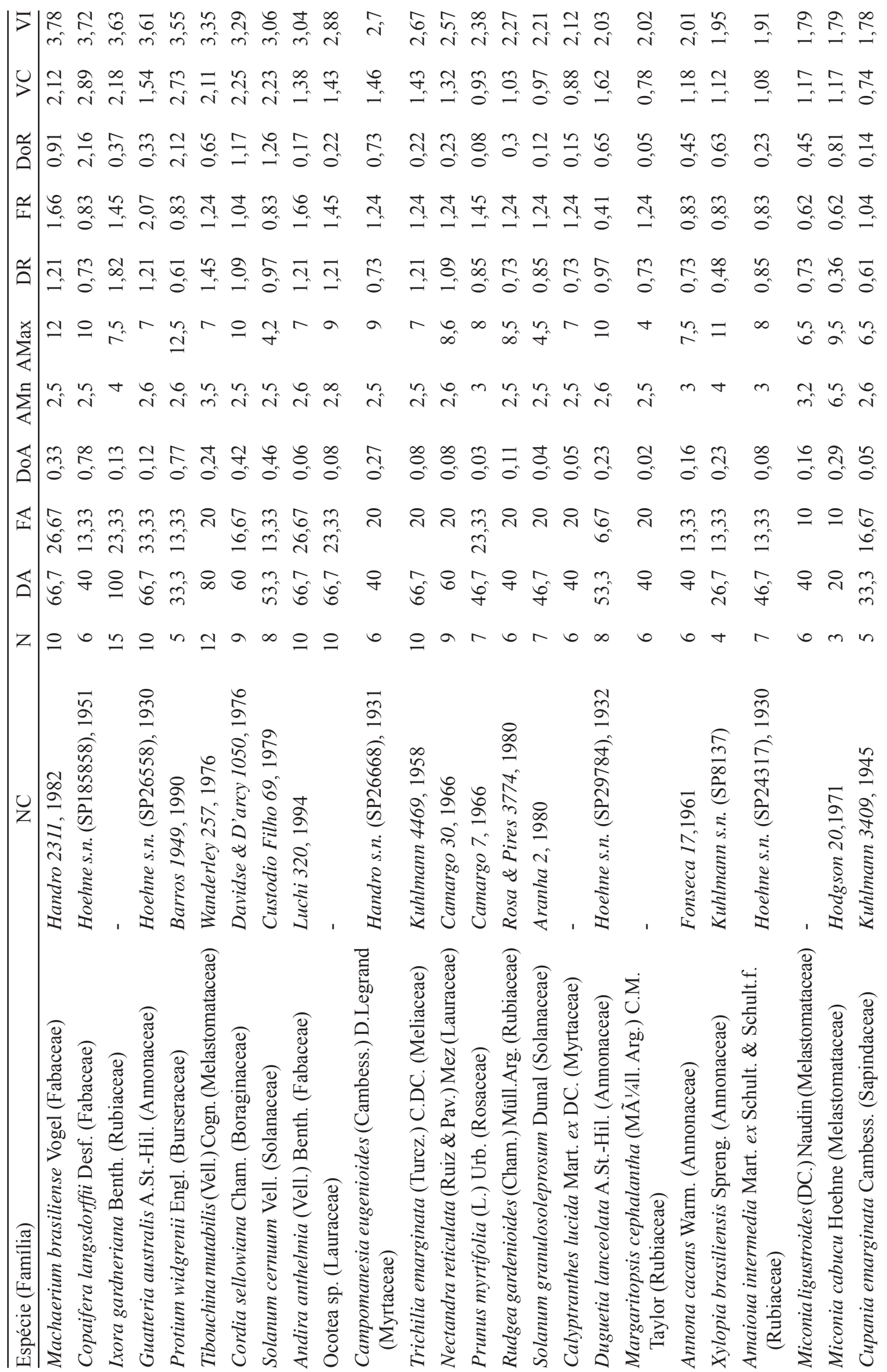




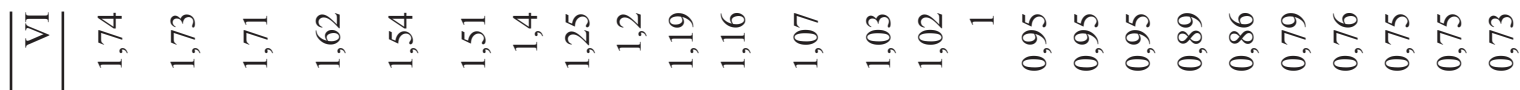

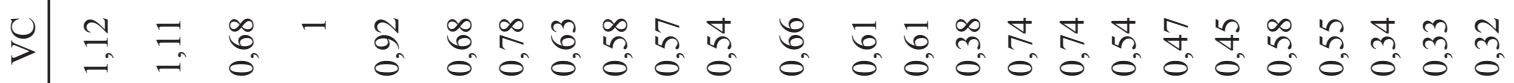

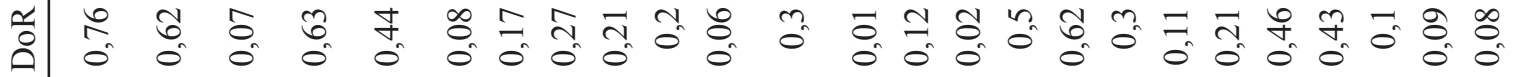

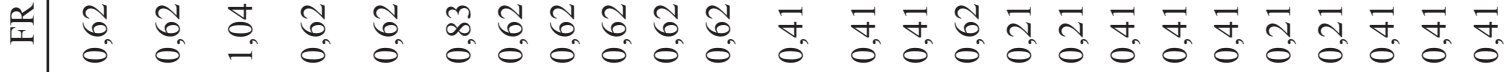

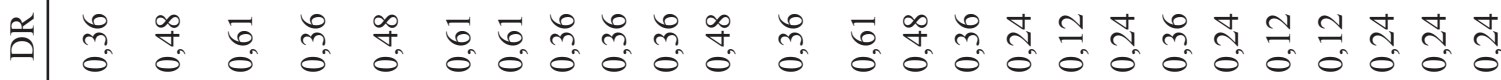

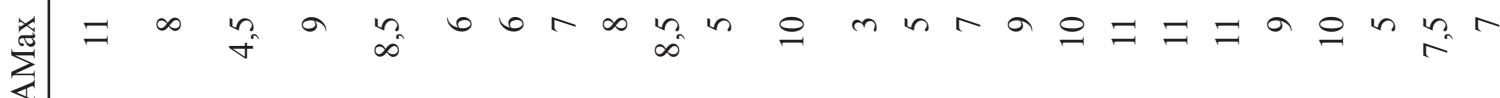

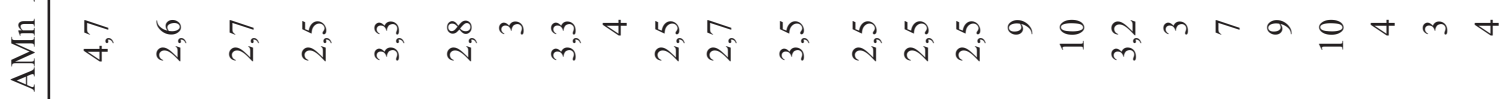

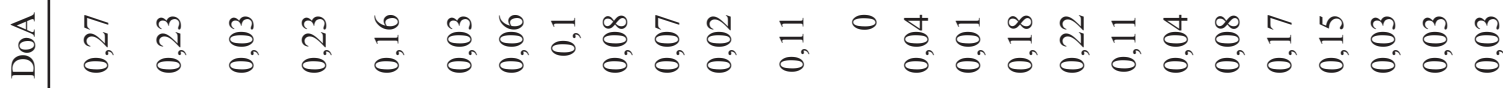

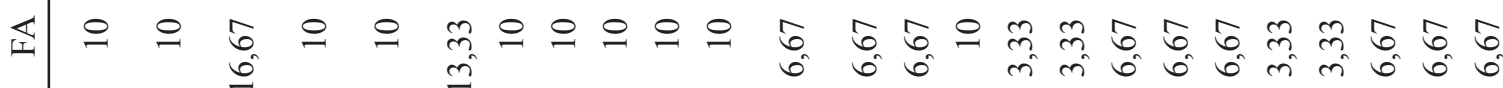

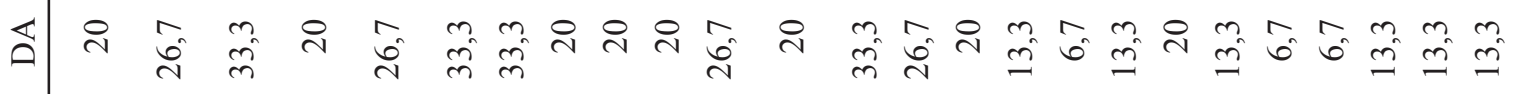

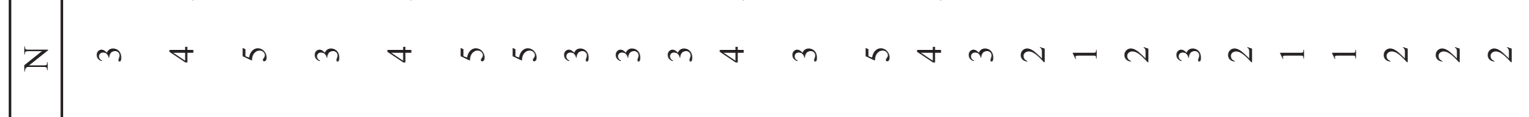

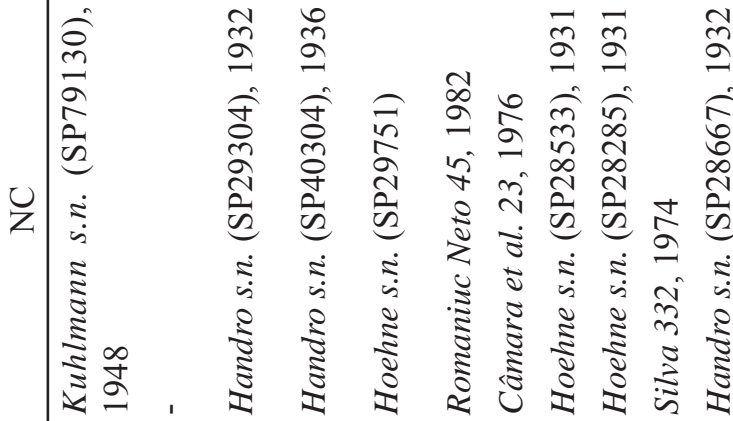

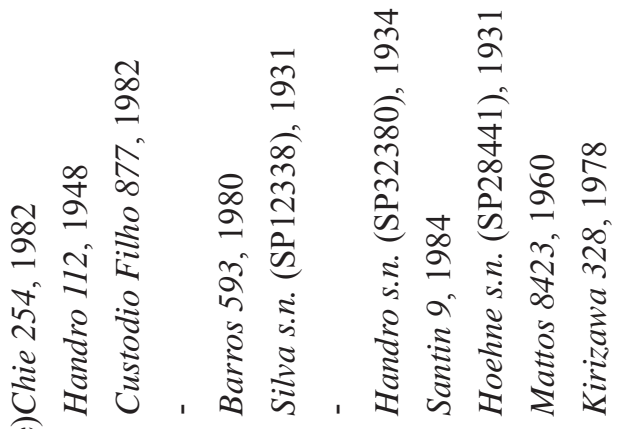

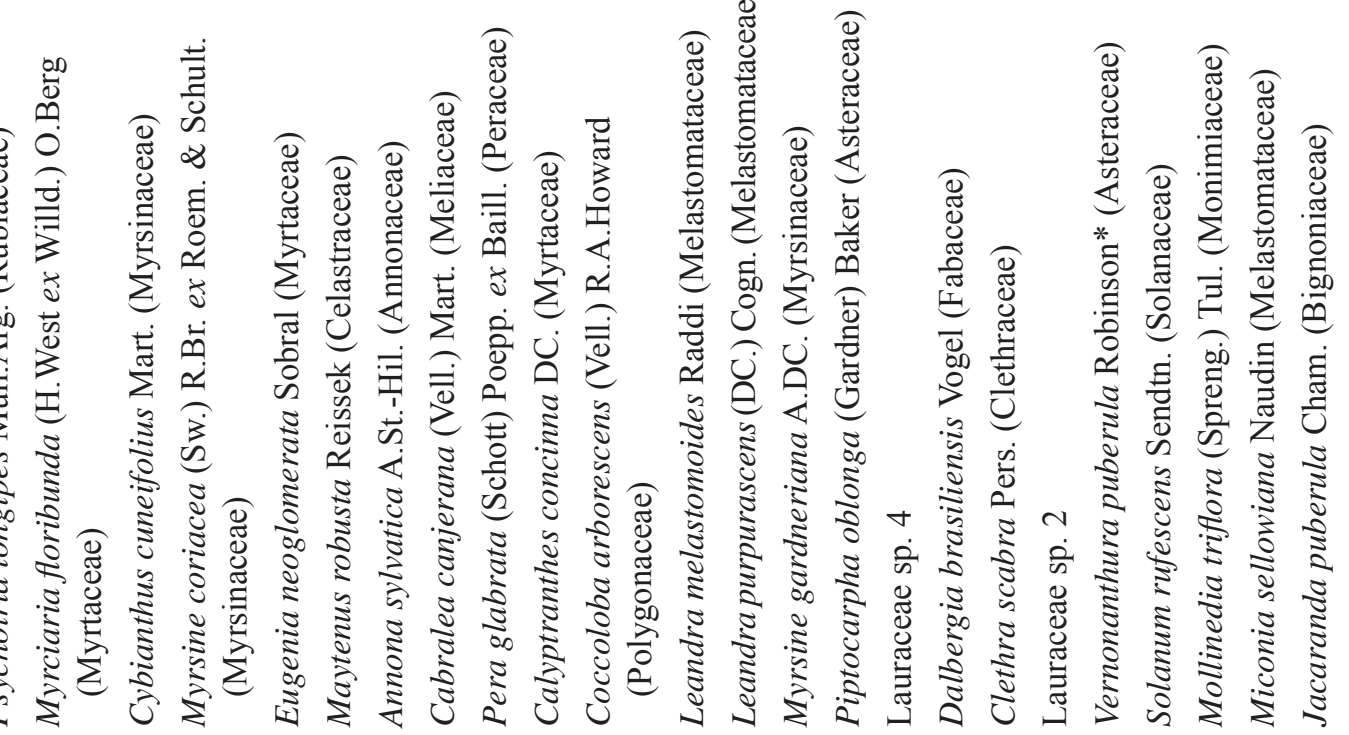




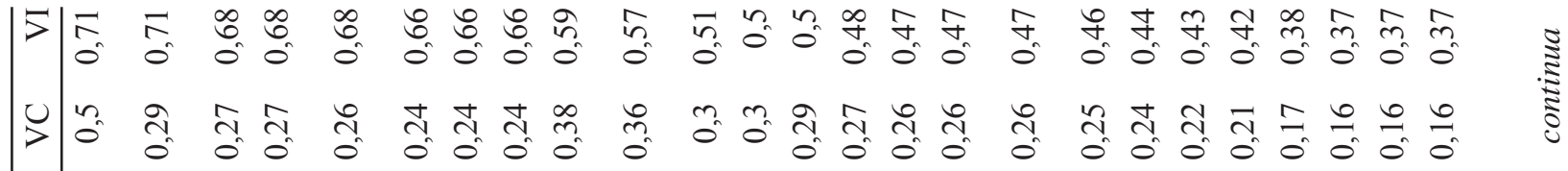

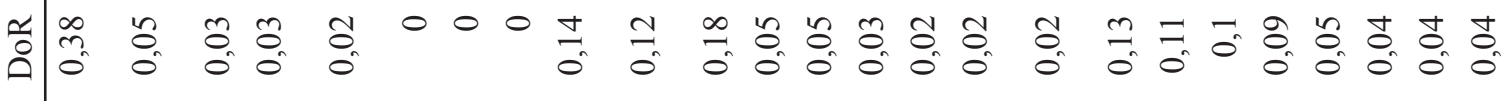

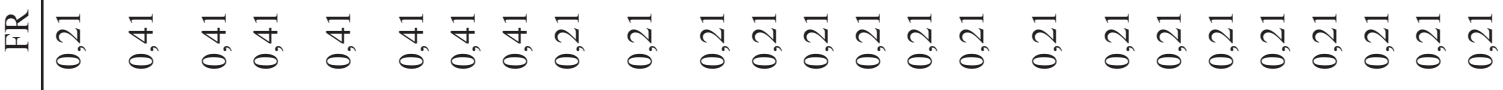

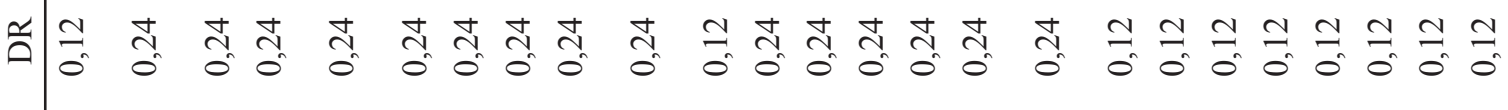

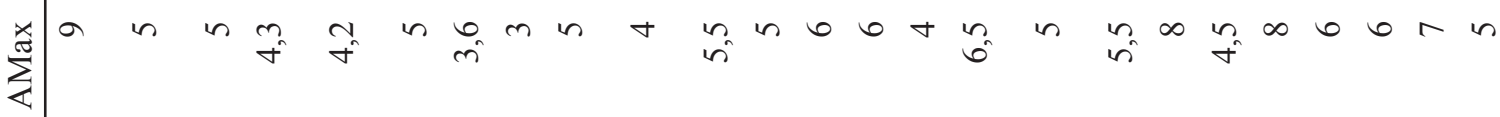

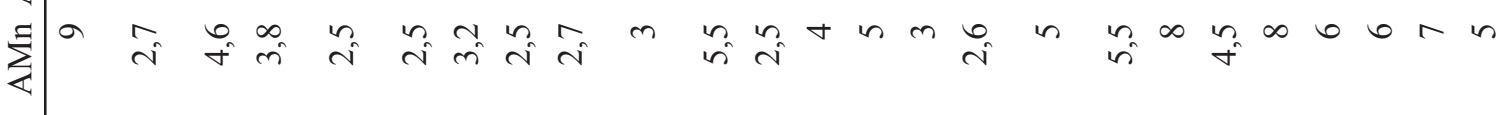

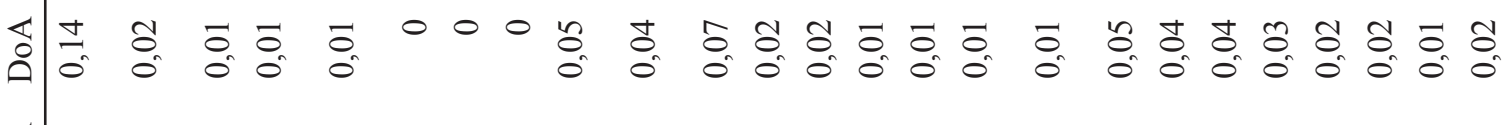

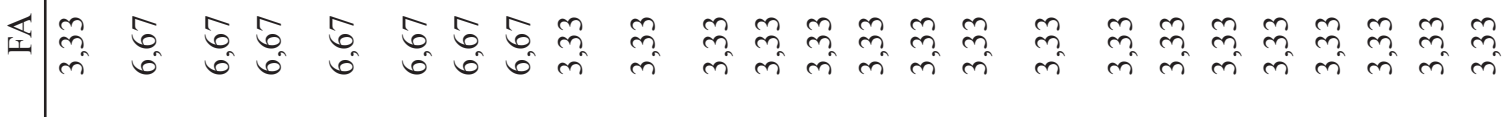

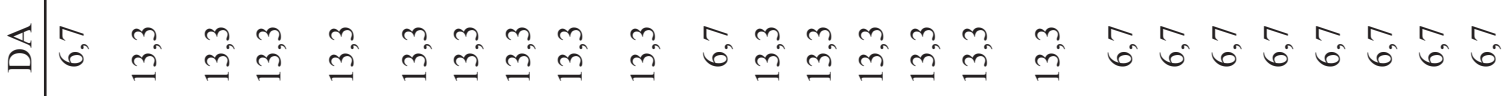

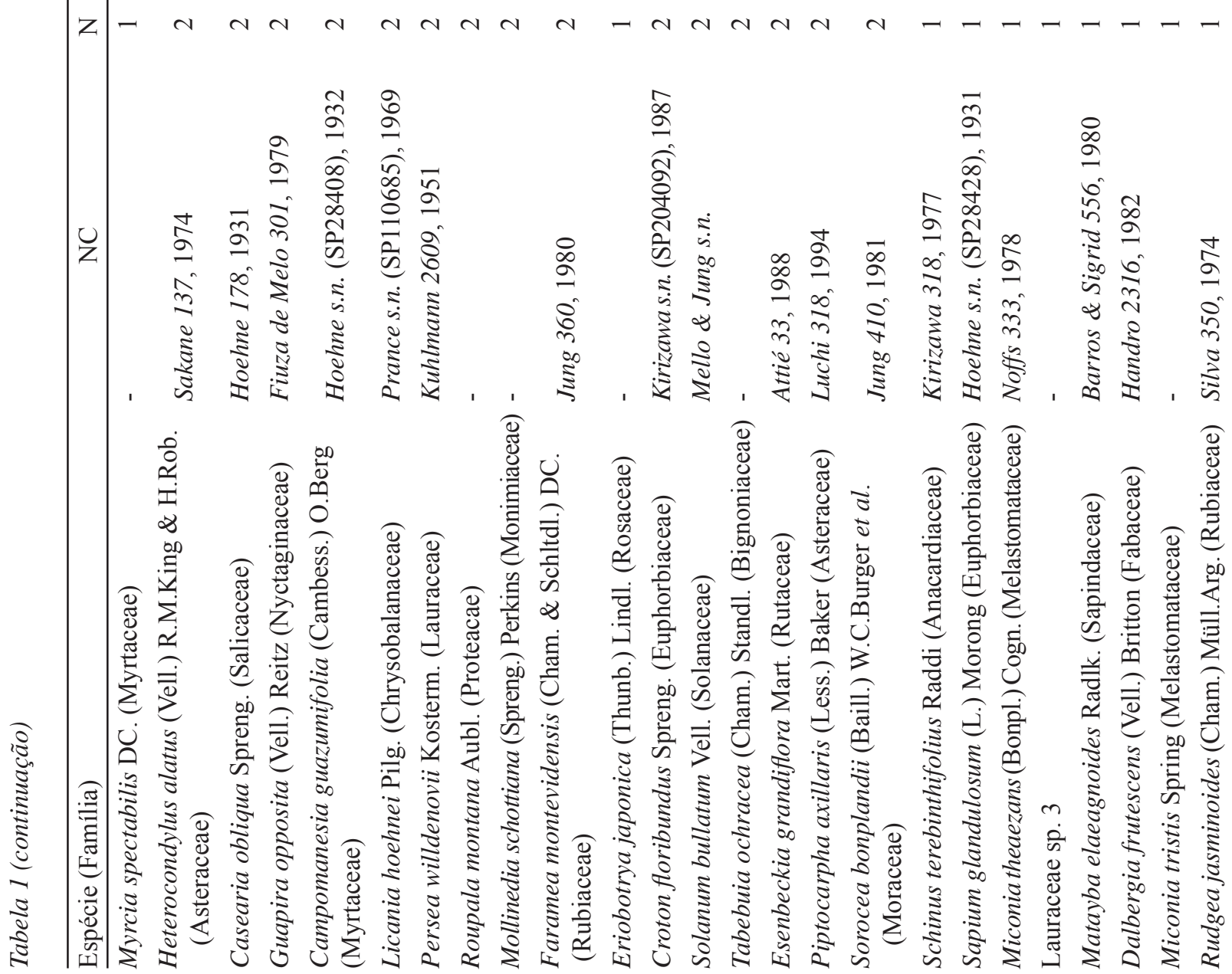



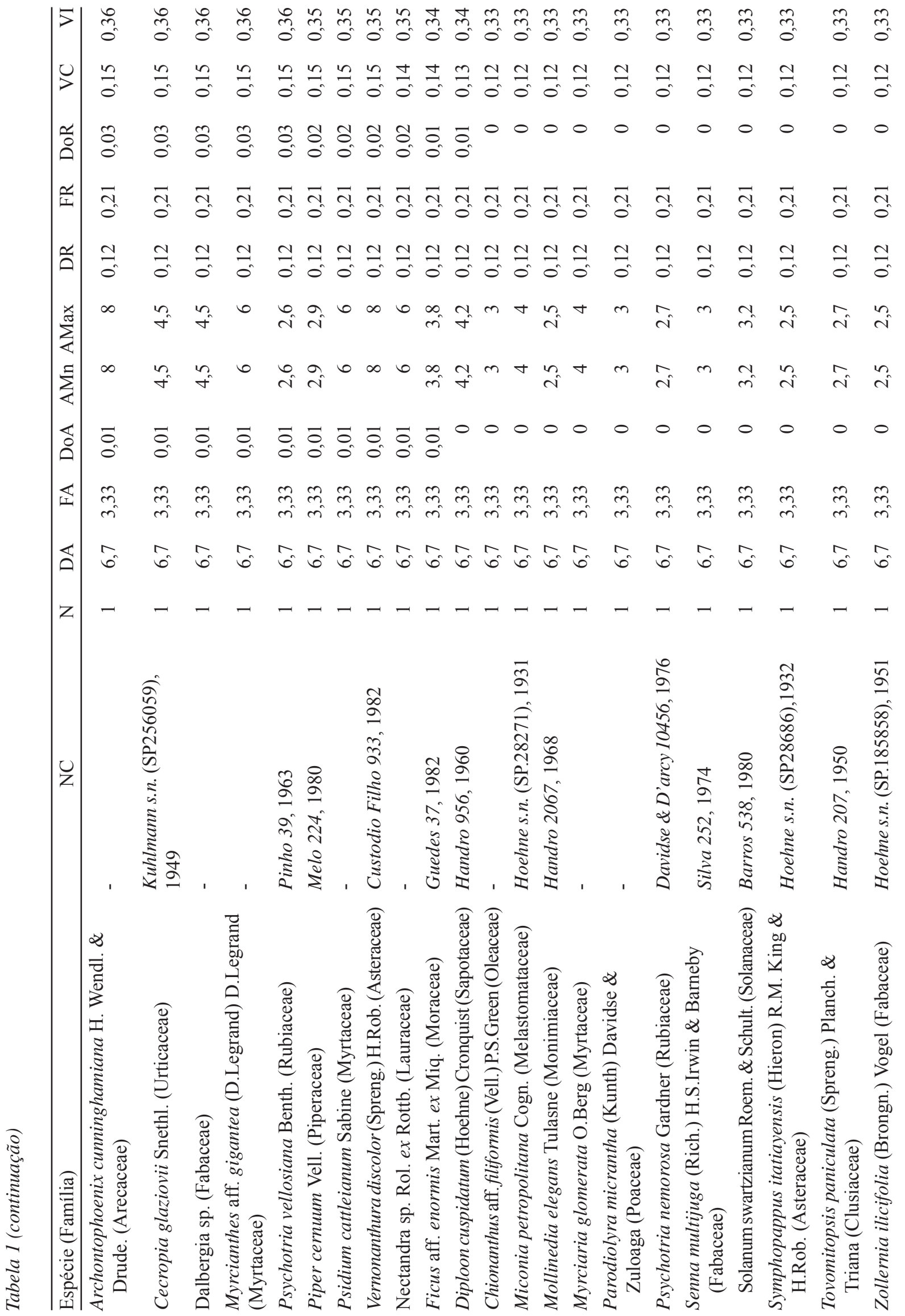
de Salles Oliveira (CUASO) (Dislich et al. 2001) para indivíduos com DAP $\geq 3,2 \mathrm{~cm}$ e densidade de 324 indivíduos ha-1 o número de espécies foi de 50 . Estas diferenças encontradas nas áreas citadas podem ser explicadas, em parte, devido ao histórico de ocupação dos locais e o impacto da fragmentação e da intensidade do efeito de borda (Rodrigues \& Nascimento 2006).

A área basal registrada $\left(31,71 \mathrm{~m}^{2} \mathrm{ha}^{-1}\right)$ pode ser considerada alta em relação aos valores encontrados em outros trechos de florestas do PEFI (Struffaldi-De-Vuono 1985 - Área A = 21,06 m² ha ${ }^{1}$ ), em áreas de floresta do município de São Paulo (Dislich et al. 2001 =20,10 $\mathrm{m}^{2} \mathrm{ha}^{-1}$ ) e trechos florestais da Grande São Paulo, como no Parque CEMUCAM, em Cotia (Ogata \& Gomes $2006=17,6 \mathrm{~m}^{2} \mathrm{ha}^{-1}$ ). No entanto de modo geral, está dentro dos valores observados em trabalhos realizados no Alto da Bacia do Tietê e áreas próximas, que possuem média da área basal aproximada de $28 \mathrm{~m}^{2} \mathrm{ha}^{-1}$, como observado em Ogata \& Gomes (2006).

Baixos valores de área basal têm sido associados à ocorrência de perturbações (Silva \& Nascimento 2001), porém, o elevado valor de área basal encontrado, pode ser reflexo da presença de espécies, como Alchornea sidifolia, a espécie com IVI mais alto $(13,19 \%$ do total) que também exibiu a maior frequência e maior densidade relativas. A. sidifolia é espécie tipicamente pioneira e heliófila (Gomes et al. 2003), sendo quase inexistente em ambientes climácicos; como espécie pioneira, possui característica de crescimento rápido, tanto em altura como em espessura (Reitz et al. 1978), o que pode ter influenciado no cômputo do diâmetro, densidade e consequentemente na área basal. Já Syagrus romanzoffiana (Cham.) Glassman com o terceiro maior IVI $(5,4 \%$ do total) evidencia que palmeiras são frequentes em locais perturbados, como o PEFI (Nastri et al. 1992, Knobel 1995, Gomes \& Mantovani 2001). Apesar desses valores, a equabilidade encontrada $(J=0,85)$ reflete a heterogeneidade da comunidade vegetal avaliada, de modo que as espécies mais dominantes apresentam baixa concentração de abundância relativa (Souza et al. 2003).

O valor de $46 \%$ para espécies com um único indivíduo, quando considerado apenas os indivíduos com $\mathrm{DAP} \geq 4,7 \mathrm{~cm}$, indica alto número de espécies raras na área de estudo. O mesmo acontece quando se compara com os demais valores, de outras regiões próximas (Grombone et al. 1990, Cardoso-Leite 1995, Ogata \& Gomes 2006). Contudo, a proporção de espécies raras em uma comunidade vegetal está diretamente relacionada com a riqueza florística do local (Silva et al. 1992). Dessa forma, a alta porcentagem de espécies raras encontrada pode explicar a alta riqueza florística observada nesse trecho do PEFI.

Essa raridade pode ter influência sobre a persistência e manutenção das espécies, pois em fragmentos isolados, como o PEFI, que é uma ilha de floresta cercada pela malha urbana, a população local será tão diminuta que as chances de sobrevivência das espécies raras são poucas, em longo prazo (Laurence \& Vasconcelos 1999).

A dominância de A. sidifolia, S. romanzoffiana e a densidade relativa de lianas são indicativos de que a área de floresta estudada se encontra em estágio secundário inicial de sucessão, em decorrência de perturbações antrópicas e da fragmentação dos remanescentes. Ainda assim, esse remanescente apresenta considerável riqueza e diversidade florística, levando em consideração sua localização urbana. Apesar da área do PEFI estar classificada como Unidade de Conservação, medidas concretas se fazem necessárias, como o desenvolvimento de estudos sobre a dinâmica das populações arbóreas, em especial as espécies raras e pesquisas sobre o impacto do efeito de borda em pequenos fragmentos, visando à sua recuperação.

\section{Literatura citada}

Angiosperm Phylogeny Group [APG]. 2009. An update of the Angiosperm Phylogeny Group classification for the orders and families of flowering plants: APG III. Botanical Journal of the Linnean Society 161: 105-121.

Aragaki, S. 1997. Florística e estrutura de trecho remanescente de floresta no planalto paulistano (SP). Dissertação de Mestrado, Universidade de São Paulo, São Paulo.

Barbosa, L.M., Potomati, A. \& Peccinini, A.A. 2002. O PEFI: Histórico e Legislação. In: D.C. Bicudo; M.C. Forti, \& C.E.M. Bicudo (orgs.). Parque Estadual das Fontes do Ipiranga: unidade de conservação que resiste à urbanização de São Paulo. São Paulo, Secretaria do Meio Ambiente de São Paulo, pp. 15-28.

Barros, F., Melo, M.M.R.F., Chiea, S.A.C., Kirizawa, M., Wanderley, M.G.L. \& Jung-Mendaçolli, S.L. 1991. Caracterização geral da vegetação e listagem das espécies ocorrentes. In: M.M.R.F. Melo, Barros, F., Wanderley, M.G.L., Kirizawa, M., Jung-Mendaçolli, S.L. \& Chiea, S.A.C. (ed.). Flora Fanerogâmica da Ilha do Cardoso Instituto de Botânica, Instituto de Botânica, São Paulo, pp. 27-184. 
Barros, F., Mamede, M.C.H., Melo, M.M.R.F., Lopes. E.A., Jung-Mendaçolli, S.L., Kirizawa, M., Muniz, C.F.S., Makino-Watanabe, H., Chiea, S.A.C. \& Melhem, T.S. 2002. A flora fanerogâmica do PEFI: composição, afinidades e conservação. In: D.C. Bicudo, M.C. Forti \& C.E.M. Bicudo (orgs.). Parque Estadual das Fontes do Ipiranga (PEFI): unidade de conservação que resiste à urbanização de São Paulo. Secretaria do Meio Ambiente do Estado de São Paulo, São Paulo, pp. 93-110.

Cardoso-Leite, E. 1995. Ecologia de um fragmento florestal em São Roque, SP: florística, fitossociologia e Silvigênese. Dissertação de Mestrado, Universidade Estadual de Campinas, Campinas.

Dislich, R., Cersósimo, L. \& Mantovani, W. 2001. Análise da estrutura de fragmentos florestais no Planalto Paulistano - SP. Revista Brasileira de Botânica 24: 321-332.

Felfili, J.M. \& Rezende, R.P. 2003. Conceitos e métodos em fitossociologia. Universidade de Brasília, Brasília.

Fernandes, A.J., Reis, L.A.M. \& Carvalho, A. 2002. Caracterização do meio físico. In: D.C. Bicudo; M.C. Forti \& C.E.M. Bicudo (orgs.). Parque Estadual das Fontes do Ipiranga: unidade de conservação que resiste à urbanização de São Paulo. Secretaria do Meio Ambiente de São Paulo, São Paulo, pp. 49-62.

Forzza, R.C., Leitman, P.M., Costa, A.F., Carvalho Jr, A.A., Peixoto, A.L., Walter, B.M.T., Bicudo, C., Zappi, D., Costa, D.P., Lleras, E., Martinelli, G., Lima, H.C., Prado, J., Stehmann, J.R., Baumgratz, J.F.A., Pirani, J.R., Sylvestre, L., Maia, L.C., Lohmann, L.G., Queiroz, L.P., Silveira, M., Coelho, M.N., Mamede, M.C., Bastos, M.N.C., Morim, M.P., Barbosa, M.R., Menezes, M., Hopkins, M., Secco, R., Cavalcanti, T.B. \& Souza, V.C. 2010. Introdução. In: Lista de Espécies da Flora do Brasil. Jardim Botânico do Rio de Janeiro, pp. 21-60.

Gentry, A.H. 1988. Changes in plant community diversity and floristic composition on environmental and geographical gradients. Annals of the Missouri Botanical Garden 75: 1-34.

Gomes, E.P.C. 1992. Estrutura do componente arbóreo de um trecho de mata em São Paulo, SP. Dissertação de Mestrado, Universidade de São Paulo, São Paulo.

Gomes, E.P.C. 1998. Dinâmica do componente arbóreo de um trecho de mata em São Paulo, SP. Tese de Doutorado, Universidade de São Paulo, São Paulo.

Gomes, E.P.C. \& Mantovani, W. 2001. Size structure of six tree populations in a subtropical rain forest in southeastern Brazil. Naturalia 26: 131-158.

Gomes, E.P.C., Mantovani, W. \& Kageyama, P.Y. 2003. Mortality and recruitment of trees in a secondary montane rain forest in southeastern Brazil. Brazilian Journal of Biology 63: 35-45
Grombone, M.T., Bernacci, L.C., Meira Neto, J.A.A., Tamashiro, J.Y. \& Leitão Filho, H.F. 1990. Estrutura fitossociológica da floresta semidecídua de altitude do Parque Municipal da Grota Funda (Atibaia - Estado de São Paulo). Acta Botanica Brasilica 4: 47-64.

Joly, C.A., Leitão Filho, H.F. \& Silva, S.M. 1991. O patrimônio florístico - The floristic heritage. In: G.I. Câmara (coord.). Mata Atlântica - Atlantic rain forest. Fundação S.O.S. Mata Atlântica, São Paulo, pp. 94-125.

Knobel, M.G. 1995. Aspectos da regeneração natural do componente arbóreo-arbustivo, de trecho da floresta da Reserva Biológica do Instituto de Botânica em São Paulo, SP. Dissertação de Mestrado, Universidade de São Paulo, São Paulo.

Laurance, W. \& Vasconcelos, H. 2009. Consequências Ecológicas da Fragmentação Florestal na Amazônia. Oecologia Brasiliensis 13: 434-451.

Leitão Filho, H.F. 1992. A flora arbórea dos cerrados do Estado de São Paulo. Hoehnea 19: 151-163.

Mamede, M.C.H., Cordeiro, I. \& Rossi, L. 2001. Flora vascular da Serra da Juréia, Município de Iguape, São Paulo, Brasil. Boletim do Instituto de Botânica 15: 63-124.

Martins, F.R. 1991. Estrutura de uma floresta mesófila. Editora da UNICAMP, Campinas.

Melhem , T.S., Giulietti, A.M., Forero, E., Barroso, G.M., Silvestre, M.S.F., Jung, S.L., Makino, H., Melo, M.M.R.F., Chiea, S.C., Wanderley, M.G.L., Kirizawa, M. \& Muniz, C. 1981. Planejamento para a elaboração da "Flora Fanerogâmica da Reserva do Parque Estadual das Fontes do Ipiranga (São Paulo, Brasil)". Hoehnea 9: 63-74.

Michalski, F., Nishi, I. \& Peres, C.A. 2007. Disturbancemediated drift in tree functional groups in Amazonian forest fragments. Biotropica 39: 691-701.

Mueller-Dumbois, D. \& Ellenberg, H. 1974. Aims and methods of vegetation ecology. John Wiley \& Sons, New York.

Nascimento, H.E.M. \& Laurance, W.F. 2006. Efeitos de área e de borda sobre a estrutura florestal em fragmentos de floresta de terra-firme após 13-17 anos de isolamento. Acta Amazonica 36: 183-192.

Nastri, V.D.F., Catharino, E.L.M., Rossi, L., Barbosa, L.M., Pirré, E., Bedinelli, C., Asperti, L.M., Dorta, R. de O. \& da Costa, M.P. 1992. Estudos fitossociológicos em uma área do Instituto de Botânica de São Paulo utilizados em programa de educação ambiental. Revista do Instituto Florestal 4: 219-225.

Ogata, H. \& Gomes, E.P.C. 2006. Estrutura e composição da vegetação no Parque CEMUCAM, Cotia, SP. Hoehnea 33: 371-384.

Peccinini, A.A. \& Pivello, V.R. 2002. Histórico do uso das terras e condição da vegetação no PEFI. In: D.C. Bicudo; M.C. Forti \& C.E.M. Bicudo (orgs.). Parque Estadual das Fontes do Ipiranga: unidade de conservação que resiste à urbanização de São Paulo. Secretaria do Meio Ambiente de São Paulo, São Paulo, pp. 251-258. 
Pivello, V.R. \& Peccinini, A.A. 2002. A vegetação do PEFI. In: D.C. Bicudo; M.C. Forti, \& C.E.M. Bicudo (orgs.). Parque Estadual das Fontes do Ipiranga: unidade de conservação que resiste à urbanização de São Paulo. Secretaria do Meio Ambiente de São Paulo, São Paulo, pp. 15-28.

Reitz, R., Klein, R.M. \& Reis, A. 1978. Projeto madeira de Santa Catarina. Sellowia 28-30: 218-224.

Rodrigues, P.J.F.P. \& Nascimento, M.T. 2006. Fragmentação florestal: breves considerações teóricas sobre efeitos de borda. Rodriguésia 57: 63-74.

Rossi, L. 1994. A flora arbóreo-arbustiva da mata da Reserva da Cidade Universitária "Armando de Salles Oliveira" (São Paulo, Brasil). Boletim do Instituto de Botânica 9: 1-105.

Santin,D.A. 1999. Avegetação remanescente do município de Campinas (SP): mapeamento, caracterização fisionômica e florística, visando a conservação. Tese de Doutorado, Universidade Estadual de Campinas, Campinas.

Santos, P.M. \& Funari, F.L. 2002. Clima local. In: D.C. Bicudo; M.C. Forti, \& C.E.M. Bicudo (orgs.). Parque Estadual das Fontes do Ipiranga: unidade de conservação que resiste à urbanização de São Paulo. Secretaria do Meio Ambiente do Estado de São Paulo, São Paulo, pp. 29-48.

Shepherd, G.J. 2010. Fitopac - Manual do usuário. Universidade Estadual de Campinas, Campinas.

Silva, A.S.L., Lisboa, P.L.B. \& Maciel, U.N. 1992. Diversidade florística e estrutura em floresta densa da bacia do rio Juruá-AM. Boletim do Museu Paraense Emílio Goeldi, Série Botânica 8: 203-258.

Silva, G.C. \& Nascimento, M.T. 2001. Fitossociologia de um remanescente de mata sobre tabuleiros no norte do estado do Rio de Janeiro (Mata do Carvão). Revista Brasileira de Botânica 24: 51-62.
Souza, J.S., Espírito-Santo, F.D.B., Fontes, M.A.L., Oliveira-Filho, A.T. \& Botezelli, L. 2003. Análise das variações florísticas e estruturais da comunidade arbórea de um fragmento de Floresta Semidecídua às margens do rio Capivari, Lavras-MG. Revista Árvore 27: 185-206.

Struffaldi-de-Vuono, Y.S. 1985. Fitossociologia do estrato arbóreo da floresta da Reserva Biológica do Instituto de Botânica (São Paulo-SP). Tese de Doutorado, Universidade de São Paulo, São Paulo.

Tabarelli, M. \& Mantovani, W. 1997. Colonização de clareiras naturais na floresta Atlântica no Sudeste do Brasil. Revista Brasileira de Botânica 20: 57-66.

Tabarelli, M. \& Mantovani, W. 1999. Clareiras naturais e a riqueza de espécies pioneiras em uma Floresta Atlântica Montana. Revista Brasileira de Biologia 59: 251-261.

Tanus, M.R. 2011. Florística e estrutura da comunidade arbóreo-arbustiva e o efeito de borda em trecho de Mata Atlântica no Parque Estadual das Fontes do Ipiranga, São Paulo, SP. Dissertação de Mestrado, Instituto de Botânica, São Paulo.

Thomas, W.W., Carvalho, A.M., Amorim, A.M.A., Garrison, J. \& Arbeláez, A.L. 1998. Plant endemism in two forests in southern Bahia, Brasil. Biodiversity and Conservation 7: 311-322.

Turner, I.M. \& Corlett, R.T. 1996. The conservation value of small isolated fragments of lowland tropical rain forest. Trends in Ecology and Evolution 11: 330-333.

Viana, V.M., Tabanez, A.A.J. \& Batista, J.L. 1997. Restoration and management of fragmented landscapes. In: W. F. Laurance \& R. O. Bierregaard (eds.). Tropical forest remnants: ecology, management, and conservation of fragmented communities. University of Chicago Press, Chicago, pp. 347-365. 